\title{
El impacto de la crisis argentina en el bienestar de los hogares
}

\section{Ariel Fiszbein}

Banco Mundial

afiszbein@worldbank.org

\section{Paula Inés Giovagnoli \\ Banco Mundial \\ pgiovagnoli@worldbank.org \\ Isidro Adúriz \\ Opinión Pública \\ Servicios y Mercados \\ (OPMS) \\ iaduriz@hotmail.com}

Este trabajo presenta los resultados iniciales de una encuesta de hogares realizada para evaluar los efectos sobre el bienestar de la crisis económica en Argentina. Asimismo, se pretende identificar los mecanismos y estrategias de supervivencia adoptados por los hogares durante estos tiempos difíciles. Los resultados obtenidos confirman la imagen negativa reflejada en los indicadores macroeconómicos, dan cuenta de las limitaciones en la eficacia de los distintos mecanismos de supervivencia y muestran efectos serios sobre el bienestar. La evidencia sugiere que tales efectos son más marcados en el uso de los servicios de salud que en la educación. 


\section{I}

\section{Introducción}

Argentina se encuentra actualmente sumida en una profunda crisis económica, social y política sin paralelo. Después de tres años de recesión ininterrumpida, la crisis económica y financiera se profundizó durante el año 2001. En el transcurso de dicho año se hicieron varios intentos infructuosos por reactivar el crecimiento como preludio para mejorar las finanzas públicas y el perfil de la deuda. Los esfuerzos por proteger el sistema bancario frente al amplio retiro de los depósitos (con el "corralito") tuvieron como resultado restringir la liquidez y la actividad económica y generaron descontento popular. El proceso culminó con la renuncia del Presidente De la Rúa en diciembre de 2001 y con una rápida sucesión de presidentes designados, el anuncio formal de la cesación de pagos sobre la deuda del sector público y, a principios de 2002, el abandono del Plan de Convertibilidad.

Con una recesión que ya lleva cuatro años, se ha proyectado que en 2002 la economía declinaría alrededor del $16 \%$, lo que significa que la caída del PIB sería de más del 25\% desde su mayor nivel en 1998. Con una marcada depreciación del peso desde su flotación y una política monetaria indefinida, Argentina está experimentando una significativa inflación por primera vez desde 1991. Entre la declinación de la actividad económica y la depreciación de la moneda, el PIB per cápita se derrumbó abruptamente, a una cifra proyectada de 2850 dólares en 2002 (comparado con 8210 dólares en su más alto nivel de 1998).

La caída de la última mitad de 2002 agravó severamente las ya difíciles condiciones sociales del país. Los niveles de desempleo, que ya eran elevados (18.3\% en octubre de 2001), saltaron al $21.5 \%$ según datos de

\footnotetext{
$\square$ Este trabajo fue elaborado y publicado como documento informal en la serie Documentos de trabajo por la Oficina del Banco Mundial para Argentina, Chile, Paraguay y Uruguay. Los autores agradecen a Diane Steele, Esteban Font Guido, Miriam García, Irene Novakosky y al equipo del sIEMPro, Sebastián Galiani, Sandra Cesilini, Kinnon Scott, Susana Aparicio, Polly Jones, Martín Ravallion, Florencia Castro Leal y Emanuela Galasso por su útil ayuda en la encuesta. También agradecen a Truman Packard, Indermit Gill, Leonardo Gasparini, Guillermo Cruces, Carlos Cavalcanti, Juan Gaviria, Carlos Sobrado, Estanislao Gacitúa y Chris Chamberlin por sus comentarios y sugerencias y a Enrique Zuleta Puceiro por su guía para el desarrollo e implementación de la encuesta. Las opiniones aquí expresadas corresponden a los autores y no deben ser atribuidas al Banco Mundial.
}

mayo de 2002, de acuerdo con los indicadores oficiales. ${ }^{1}$ Además, la destrucción de puestos de trabajo ha sido importante (afectando particularmente a los empleos no calificados). Durante el primer trimestre de 2002 la industria de la construcción, que es clave para la mano de obra no calificada, cayó $25 \%$ con respecto al último trimestre de 2001 (42\% en comparación con el primer trimestre de 2001). ${ }^{2}$ También la pobreza está en aumento, confirmando la asociación entre la crisis económica y el incremento de la pobreza (Lustig, 2000). Las estimaciones oficiales para 28 centros urbanos indican que aumentó del $38.3 \%$ en octubre de 2001 a $53.0 \%$ en mayo de 2002. Este contexto extremadamente negativo también ha tenido un fuerte impacto sobre los sectores de la salud y la educación, en los que hay creciente evidencia de deterioro en la provisión de servicios. El efecto combinado de todos estos factores ha llevado a una situación social cada vez más conflictiva, con altos niveles de delito, violencia y diversas formas de protesta.

En este trabajo se presentan los resultados iniciales de una encuesta de hogares especialmente realizada para evaluar los efectos que ha tenido la crisis económica sobre el bienestar de los hogares e identificar los mecanismos y estrategias de supervivencia adoptados por las familias argentinas durante estos tiempos difíciles. Específicamente, la encuesta fue diseñada para identificar cambios en los ingresos, los patrones de consumo, el acceso a servicios sociales y otros, los patrones de ahorro y de pago, la participación en el mercado laboral y la dependencia de mecanismos de contención social formales e informales. Con ella se quiso, además, recabar información sobre los cambios en el estado mental y emocional de las personas.

La encuesta es representativa a nivel nacional, y cubrió 2800 hogares de diferentes regiones. ${ }^{3}$ A diferencia de la mayoría de las encuestas de Argentina, incluida la Encuesta Permanente de Hogares (EPH) del INDEC, ésta incluyó pequeñas localidades (con menos de 2000

\footnotetext{
${ }^{1}$ Instituto Nacional de Estadísticas y Censos (INDEC), Encuesta Permanente de Hogares (EPH), total áreas urbanas.

${ }^{2}$ Encuesta de Indicadores Laborales (EIL), Ministerio de Trabajo.

${ }^{3}$ La encuesta fue realizada por "Opinión Pública Servicios y Mercados" (OPMS).
} 
habitantes), de modo de contar con un mejor conocimiento de esas áreas. No obstante, la encuesta no incluyó las áreas dispersas y, por lo tanto, no presenta un cuadro completo del heterogéneo sector rural argentino. En el caso de las áreas urbanas, el diseño de la muestra se basó en una estratificación por tamaño de ciudad y por región. Para las zonas rurales se seleccionaron las localidades en forma aleatoria. Si bien se hizo el esfuerzo de incluir localidades de diferentes regiones, la muestra no es lo suficientemente grande como para ser representativa a nivel regional. En términos generales, el diseño de la muestra permite las comparaciones con la EPH de las áreas urbanas.

La primera parte del cuestionario solicita información sobre todos los miembros del hogar e incluye preguntas sobre características demográficas, empleo, ingresos, estado migratorio, nivel de educación, cobertura médica y aportes a la seguridad social. Varias de estas preguntas son retrospectivas, usando el mes de octubre de 2001 como punto de referencia. Se preguntó a los encuestados sobre sus ingresos individuales laborales y no laborales (en efectivo y en especie), así como sobre las transferencias de ingresos oficiales y no oficiales.

La segunda parte del cuestionario se concentró en el hogar como unidad de análisis y el respondente fue el jefe de hogar (o la persona mejor informada del hogar). ${ }^{4}$ Incluyó preguntas sobre el uso de ahorros, cambios en los patrones de consumo, y participación en programas sociales y actividades comunitarias. También se preguntó sobre el estado mental y emocional y las expectativas para el futuro del respondente. El cuestionario difería levemente en el caso de las áreas rurales y en el de las urbanas a fin de captar las especificidades correspondientes. El trabajo de campo se realizó en junio y julio de 2002.

\section{II}

\section{Principales hallazgos}

\section{Ingreso de los hogares}

En el cuadro 1 se presenta la información básica sobre ingresos derivada de la encuesta. Los ingresos mensuales per cápita informados, provenientes de todas las fuentes, ${ }^{5}$ ascendieron a 214.6 pesos. Esto puede compararse con un ingreso promedio de 233 pesos estimado por el INDEC para áreas urbanas en mayo de 2002. Siguiendo los procedimientos estándares, el in-

\footnotetext{
${ }^{4} \mathrm{Si}$ el jefe de hogar no conocía la respuesta a una pregunta en particular (por ejemplo, los cambios en los patrones de consumo) se visitó el hogar hasta un máximo de tres veces para encontrar un miembro que pudiera responder a esa pregunta.

${ }^{5}$ Debido a los conocidos problemas de las encuestas a hogares en la captación de los niveles de ingreso, se consideró una pregunta para las personas que no quisieron informar su nivel exacto de ingresos, en la que se les pidió que seleccionaran el intervalo que más se aproximara su ingreso (y para imputar este ingreso se tomaron los valores medios de cada intervalo). Esto permitió reducir el número de hogares con información incompleta sobre ingresos del $17.2 \%$ al $7.6 \%$. En el caso de los individuos que no informaron los ingresos laborales, éstos se estimaron usando las ecuaciones de Mincer, donde el logaritmo del ingreso laboral horario es definido como función de los atributos individuales observables, tales como sexo, educación, edad y experiencia. Para corregir el sesgo de selección se usó el método de Heckman. Los efectos absolutos y relativos de estas correcciones sobre los ingresos son insignificantes: aumentos de ingresos per cápita de 212 a 214 pesos (Heckman, 1979).
}

CUADRO 1

Argentina: Ingreso medio por quintiles, junio de 2002

\begin{tabular}{lcccc}
\hline Quintiles & \multicolumn{2}{c}{$\begin{array}{c}\text { Ingreso del hogar } \\
\text { por adulto equivalente }(\$)\end{array}$} & \multicolumn{2}{c}{$\begin{array}{c}\text { Ingreso per cápita } \\
\text { del hogar }\end{array}$} \\
\cline { 1 - 2 } & Media (\$) & Participación (\%) & Media (\$) & Participación (\%) \\
\hline I & 40.5 & 3.1 & 32.0 & 3.0 \\
II & 107.4 & 8.1 & 85.9 & 8.0 \\
III & 182.7 & 13.8 & 146.3 & 13.7 \\
IV & 300.1 & 22.7 & 240.9 & 22.5 \\
V & 690.8 & 52.3 & 564.7 & 52.8 \\
Media & 264.3 & 100 & 214.6 & 100.0 \\
Y10/Y1 b & 28.9 & & 28.4 & \\
Coeficiente & & & & \\
de Gini & 0.473 & & 0.478 & \\
\hline
\end{tabular}

a El primer quintil incluye los hogares con ingresos cero.

b Y10/Y1 es la razón de ingresos medios correspondientes al decil superior e inferior en la distribución. Y1 no incluye a los que informaron ingresos iguales a cero.

greso per cápita fue ajustado usando la escala de adulto equivalente (para reflejar las diferencias en las necesidades de consumo), lo que arrojó un ingreso mensual promedio de 264 pesos. También se presentan algunas medidas de desigualdad estándar que confirman los niveles relativamente elevados de desigualdad 
de ingresos observados en las encuestas de hogares de Argentina.

Como se mencionó, una característica particular de esta encuesta es la inclusión de las áreas rurales, que no son cubiertas en la EPH. El cuadro 2 confirma el hecho bien conocido (pero no necesariamente bien establecido) de que los niveles de ingresos urbanos son más elevados, indicando una brecha del orden del $60 \%$.

A fin de identificar los efectos de la crisis sobre los ingresos de la familia, en la encuesta se pidió a cada hogar que indicara si sus ingresos corrientes se habían modificado - en términos nominales- con respecto a octubre de 2001. En el cuadro 3 puede verse que más del $40 \%$ de los hogares informó no haber tenido cambios en sus ingresos, mientras que casi el $50 \%$ informó una reducción en sus ingresos nominales. Cabe subrayar que alrededor del $8 \%$ de los hogares informó de aumentos en sus ingresos.

La información obtenida no permite determinar el grado de influencia de estos cambios en la distribución del ingreso, dado que las preguntas retrospectivas no hacían referencia a "los niveles" de ingreso. En el cuadro 4, sin embargo, puede verse su incidencia según el nivel de educación del jefe/jefa de hogar -un sustituto para el ingreso del hogar.

Es interesante observar que la mayor incidencia de la reducción en los niveles de ingresos corresponde a hogares cuyos jefes tienen educación secundaria - un grupo que tiende a estar ubicado en el punto medio de la distribución de ingresos ('la clase media') - mientras que la incidencia más baja de la reducción del nivel de ingresos corresponde a hogares que tienen como jefe/jefa a personas con educación primaria incompleta — un grupo que tiende a estar

CUADRO 2

Argentina: Ingreso medio por áreas (En pesos)

\begin{tabular}{lcc}
\hline Áreas & $\begin{array}{c}\text { Ingreso del hogar } \\
\text { por adulto equivalente }\end{array}$ & $\begin{array}{c}\text { Ingreso per cápita } \\
\text { del hogar }\end{array}$ \\
\hline Urbanas & 268.3 & 223.27 \\
Rurales & 168.9 & 142.13 \\
\hline
\end{tabular}

CUADRO 3

Argentina: Cambios en los ingresos

\begin{tabular}{lr}
\hline Con respecto a octubre de 2001 & $\%$ \\
\hline Aumentaron & 8.0 \\
Sin cambios & 43.4 \\
Se redujeron & 48.6 \\
\hline
\end{tabular}

CUADRO 4

Argentina: Cambios en el ingreso por nivel de educación del jefe/a de hogar

\begin{tabular}{|c|c|c|c|c|}
\hline & Aumentó & Sin cambios & Se redujo & Total \\
\hline $\begin{array}{l}\text { Educación primaria } \\
\text { incompleta }\end{array}$ & 4.25 & 50.14 & 45.61 & 100 \\
\hline Educación primaria & 7.81 & 44.85 & 47.34 & 100 \\
\hline $\begin{array}{l}\text { Educación secundaria } \\
\text { incompleta }\end{array}$ & 15.44 & 34.52 & 50.04 & 100 \\
\hline $\begin{array}{l}\text { Educación secundaria } \\
\text { Educación superior }\end{array}$ & 6.72 & 39.51 & 53.77 & 100 \\
\hline incompleta & 6.83 & 45.94 & 47.23 & 100 \\
\hline Educación superior & 8.91 & 41.24 & 49.85 & 100 \\
\hline Total & 8.07 & 43.22 & 48.71 & 100 \\
\hline
\end{tabular}

CUADRO 5 Argentina: Hogares que informaron de
aumentos en sus ingresos

\begin{tabular}{lr}
\hline Razones & $\%$ \\
\hline Más trabajadores & 15.0 \\
Cambio de empleo & 14.1 \\
Trabajó más horas & 14.4 \\
El mismo empleo/horas & 31.7 \\
Ingresos no laborales superiores & 22.3 \\
Otras & 2.5
\end{tabular}

ubicado en el extremo inferior de la distribución de ingresos ('los indigentes'). Esta información sugiere que las clases medias podrían haber sido las más seriamente afectadas por la crisis económica. ${ }^{6}$

De particular interés es el caso del $8 \%$ de los hogares que informaron haber tenido un aumento en sus ingresos con respecto a octubre de 2001, dado el desempeño extremadamente negativo del período. En el cuadro 5 se proporcionan algunas posibles razones de estos aumentos nominales.

En casi la mitad de los casos, el aumento en los ingresos informado está asociado con un cambio en las características de empleo: un nuevo trabajador en la familia (la mayoría en trabajos temporarios, informales), el cambio a un empleo mejor pagado, o más horas trabajadas. La otra mitad de los casos no muestra ningún cambio observable en las características del empleo de alguno de los miembros del hogar. En algo más del $20 \%$ de los casos es posible identificar un

\footnotetext{
${ }^{6}$ Por el contrario, durante la década de 1990, las conmociones económicas tuvieron un efecto más profundo sobre los pobres y quienes tenían los más bajos niveles educativos. Véase Banco Mundial (2000 y 2001).
} 
aumento en los ingresos no laborales como la principal fuente de cambio.

\section{Empleo}

La encuesta también abordó la situación individual en el mercado de trabajo, así como los cambios con respecto a ocupación en octubre de 2001. Los resultados indican una tasa de actividad estable (es decir, sin cambios netos en la participación en la fuerza de trabajo) cercana al $40 \%$, con un aumento en la tasa de desocupación del orden del 3\% combinado con una reducción en la tasa de empleo (como proporción de la población) de algo más del 1\%. La EPH registró un aumento similar en el índice de desempleo (del 18.3\% al $21.5 \%$ ) en 28 centros urbanos, si bien con niveles levemente distintos.

Estos valores agregados ocultan cambios significativos en el estado laboral de hogares específicos. En el cuadro 7 se presenta una visión resumida de dichos

CUADRO 6

Argentina: Empleo, mayo/junio de 2002 (Porcentajes)

\begin{tabular}{lcc}
\hline & $\begin{array}{c}\text { Mayo/junio } \\
\text { de } 2002\end{array}$ & $\begin{array}{c}\text { Octubre } \\
\text { de } 2001\end{array}$ \\
\hline Tasa de empleo & 29.9 & $31.1^{\mathrm{a}}$ \\
Tasa de actividad & 39.8 & $39.8^{\mathrm{a}}$ \\
Tasa de desocupación & 24.9 & $22.0^{\mathrm{a}}$ \\
Tasa de desocupación (EPH) & 21.5 & 18.3 \\
\hline a Sobre la base de información retrospectiva provista por los
\end{tabular}

cambios, concentrándose en el cambio de estatus ocupacional entre octubre de 2001 y la fecha de la encuesta, sobre la base de las preguntas retrospectivas.

La estabilidad de la participación en la fuerza laboral se confirma si se comparan las cifras de la categoría "inactivo" de ambos lados de la matriz. El número de personas que salen de la fuerza laboral (en su mayoría previamente desocupados) es similar al de quienes ingresan a ella (y que mayormente permanecen desempleados). Un análisis más detallado indica que en su mayoría se trata de "trabajadores secundarios" de los hogares en los que existe otro miembro ocupado.

Como era de esperar, el número de aquellos que pierden su puesto de trabajo supera al de los que lo encuentran. Como muestra el cuadro 8, el grupo de los que salen de la desocupación tiende a estar compuesto por trabajadores secundarios en una proporción mayor que el grupo de los que pierden su trabajo - indicando la presencia de algunos cambios en los roles familiares que será tema de un análisis posterior.

El tercer factor que vale la pena mencionar es un aparente deterioro en la "calidad" de los puestos de trabajo. Esto se puede observar como reflejo en el tipo de trabajos de los que obtuvieron nuevos puestos (temporarios más que permanentes, y entre los que informan trabajos permanentes, más de la mitad carentes por completo de los beneficios estándares asociados con el sector formal), y los que cambiaron de trabajo (indicando un aumento neto en la proporción de trabajos temporarios y una gran presencia de "trabajos sin beneficios" entre los que se cambiaron a puestos de trabajo permanentes). Aproximadamente el $6 \%$ de los

CUADRO 7

Argentina: Cambios en el estatus laboral

(En miles y porcentajes)

\begin{tabular}{|c|c|c|c|c|c|c|c|}
\hline & \multicolumn{7}{|c|}{ Estatus anterior } \\
\hline & & & Desocupado & Inactivo & Trabajo permanente & Trabajo temporario & Total \\
\hline \multirow{5}{*}{ 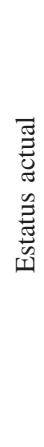 } & Desocupado & $\begin{array}{c}\text { Miles } \\
\%\end{array}$ & $\begin{array}{c}1435 \\
50.6\end{array}$ & $\begin{array}{c}1228 \\
6.2\end{array}$ & $\begin{array}{l}15 \\
0.2\end{array}$ & $\begin{array}{l}555 \\
17.1\end{array}$ & $\begin{array}{c}3233 \\
9.9\end{array}$ \\
\hline & Inactivo & $\begin{array}{c}\text { Miles } \\
\%\end{array}$ & $\begin{array}{c}1039 \\
36.7\end{array}$ & $\begin{array}{c}18252 \\
92.5\end{array}$ & $\begin{array}{l}20 \\
0.3\end{array}$ & $\begin{array}{l}386 \\
11.9\end{array}$ & $\begin{array}{c}19696 \\
60.0\end{array}$ \\
\hline & Trabajo permanente & $\begin{array}{c}\text { Miles } \\
\%\end{array}$ & $\begin{array}{l}113 \\
4.0\end{array}$ & $\begin{array}{l}91 \\
0.5\end{array}$ & $\begin{array}{c}6498 \\
92.6\end{array}$ & $\begin{array}{c}331 \\
10.2\end{array}$ & $\begin{array}{c}7034 \\
21.4\end{array}$ \\
\hline & Trabajo temporario & $\begin{array}{c}\text { Miles } \\
\%\end{array}$ & $\begin{array}{l}247 \\
8.7\end{array}$ & $\begin{array}{l}153 \\
0.8\end{array}$ & $\begin{array}{l}485 \\
6.9\end{array}$ & $\begin{array}{c}1969 \\
60.8\end{array}$ & $\begin{array}{c}2854 \\
8.7\end{array}$ \\
\hline & Total & $\begin{array}{c}\text { Miles } \\
\%\end{array}$ & $\begin{array}{c}2834 \\
100\end{array}$ & $\begin{array}{c}19725 \\
100\end{array}$ & $\begin{array}{c}7018 \\
100\end{array}$ & $\begin{array}{c}3241 \\
100\end{array}$ & $\begin{array}{c}32817 \\
100\end{array}$ \\
\hline
\end{tabular}


CUADRO 8

Argentina: Grupos que sufrieron cambios, clasificados por miembro del hogar y género

\begin{tabular}{lc}
\hline $\begin{array}{l}\text { Desocupado a } \\
\text { empleado (\%) }\end{array}$ & $\begin{array}{c}\text { Empleado a } \\
\text { desocupado (\%) }\end{array}$ \\
\hline
\end{tabular}

Miembro del hogar

$\begin{array}{lrr}\text { Jefe de hogar } & 35.9 & 57.0 \\ \text { Cónyuge } & 18.8 & 12.6 \\ \text { Otros } & 45.3 & 30.4 \\ \text { Total } & 100.0 & 100.0\end{array}$

Género

Hombre

58.4

Mujer

41.6

62.0

Total

100.0

100.0

GRAFICO 1

Gran Buenos Aires: Evolución del empleo formal

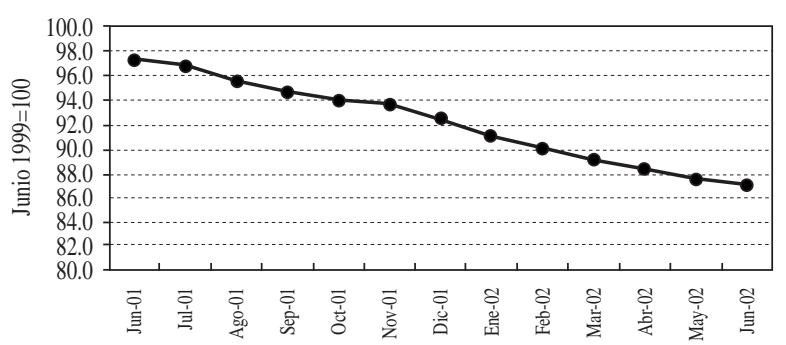

Fuente: Encuesta de Indicadores Laborales (EIL).

ocupados informó que perdió al menos algún tipo de beneficio. Esta tendencia concuerda con la observada a través de la EPH. ${ }^{7}$

El marcado deterioro en el empleo del sector formal también se confirma a través de la Encuesta de Indicadores Laborales (EIL) del Ministerio de Trabajo. Esta encuesta cubre más de 1500 empresas que representan un universo de aproximadamente 38000 firmas de diferentes tamaños y sectores, que emplean a unos 1.9 millones de trabajadores registrados en los tres centros urbanos más grandes (Gran Buenos Aires, Gran Rosario y Gran Córdoba). Como se ilustra en el gráfico 1 , se estima que el empleo formal tuvo una caída del $7.4 \%$ desde octubre de 2001, y del $10.5 \%$ desde junio de $2001 .^{8}$

\footnotetext{
${ }^{7}$ Por ejemplo, la proporción de trabajadores asalariados sin beneficios aumentó del 33\% al 35\% entre las EPH de octubre de 2001 y mayo de 2002.

${ }^{8}$ Otros datos adicionales proporcionados por la Administración Federal de Ingresos Públicos (AFIP) revelan resultados similares: el promedio mensual de puestos de trabajo en el primer trimestre de
}

\section{Pobreza}

Sobre la base de la información sobre ingresos correspondiente al mes de junio, se ha estimado la incidencia de la pobreza usando cuatro líneas diferentes. Primero, se han usado las líneas oficiales de "pobreza" e "indigencia" estimadas por el INDEC para el mes de junio de 2002. ${ }^{9}$ Para las áreas rurales, se usó la línea de pobreza correspondiente al centro urbano de la misma región. Por supuesto, de esta manera no se reconocen las potenciales diferencias entre los precios rurales y los urbanos, pero al menos se controlan las diferencias regionales (Banco Mundial, 2001). ${ }^{10}$ Además, se construyó una línea de pobreza de 1 dólar y 2 dólares por día, usando la metodología estándar del Banco Mundial para el total del país. ${ }^{11}$ Los resultados a nivel nacional se presentan en el cuadro 9 .

En el cuadro 10 se presentan los resultados para las áreas rurales y urbanas.

Conviene resaltar algunos puntos. Primero, la incidencia de la pobreza/indigencia (usando las líneas oficiales) confirma las predicciones realizadas en las primeras etapas de la crisis, que sugerían índices de pobreza del orden del $50 \%$ e índices de indigencia de alrededor de $25 \%$. Las estimaciones oficiales de la EPH revelan tasas de pobreza y de indigencia similares (53\% y $24.8 \%$, respectivamente), si bien con una cobertura más reducida dada la exclusión de las áreas rurales en esta encuesta. Segundo, con todas las consideraciones necesarias en mente, es importante observar que la línea de pobreza de 2 dólares por día es muy cercana a la línea oficial de indigencia que, en principio, brinda un mejor reflejo de cómo se compara la pobreza en la Argentina posterior a la cesación de

2002 presenta una disminución del $10.1 \%$ comparado con el mismo período del año anterior.

${ }^{9}$ Las canastas básicas de alimentos fueron originalmente estimadas para cada centro urbano sobre la base de los patrones de consumo de 1997, y se ajustaron para reflejar los precios vigentes en abril de 2001 en cada centro, usando el índice de Paridad de Precios de Compra del Consumidor (PPCC) del INDEC para dicho mes. Luego las líneas fueron actualizadas al mes de junio usando la variación de precios en el Gran Buenos Aires. En este sentido, la ausencia de información de precios sistemática para todos los centros urbanos es una seria limitación. Comparando los cambios de precios de los alimentos en Buenos Aires, Córdoba, Mendoza y Tucumán durante los primeros seis meses de 2002, se observa que el nivel de aumento es levemente más alto en el interior que en el Gran Buenos Aires.

${ }^{10} \mathrm{Se}$ realizará por separado un análisis de mayor profundidad de la pobreza en las áreas rurales.

${ }^{11}$ Se construyeron las líneas de pobreza usando el índice de Paridad de Poder Adquisitivo (PPA) para Argentina de 1993 (PPA = 0.7779) y se convirtieron a precios locales usando el índice de precios al consumidor (IPC). 
CUADRO 9

Argentina: Î́ndices de pobreza, junio de 2002

(Porcentajes de individuos)

\begin{tabular}{lccc}
\hline & $\begin{array}{c}\text { Encuesta del } \\
\text { Banco Mundial } \\
\text { Junio 2002 }\end{array}$ & \multicolumn{2}{c}{$\begin{array}{c}\text { Encuesta Permanente } \\
\text { de Hogares (EPH) }\end{array}$} \\
\cline { 3 - 4 } & & Mayo 2002 & Oct. 2001 \\
\hline Pobreza & 53.7 & 53.0 & 38.3 \\
Indigencia & 23.8 & 24.8 & 13.6 \\
PPA (1 dólar) & 9.9 & 9.5 & 7.5 \\
PPA (2 dólares) & 24.6 & 20.8 & 17.1 \\
\hline
\end{tabular}

CUADRO 10

Argentina: Índices de pobreza

por áreas, junio de 2002

(Porcentajes de individuos)

\begin{tabular}{lcc}
\hline & Urbana & Rural \\
\hline Pobreza & 52.9 & 72.6 \\
Indigencia & 23.2 & 40.1 \\
\hline
\end{tabular}

pagos con los niveles internacionales. Tercero, y nuevamente con todas las salvedades metodológicas necesarias, aun cuando la incidencia de la pobreza y la indigencia es, como se esperaba, mucho más alta en las áreas rurales, el agregado de esas áreas en la estimación no tiene un efecto significativo en las tasas nacionales, dada la magnitud relativa de los dos grupos.

\section{Bienestar subjetivo}

Con el propósito exploratorio de identificar los cambios en el estado mental y emocional de los individuos ("cómo se siente la gente"), en la encuesta se preguntaba si "se sentían desalentados" (" ¿se siente desanimado?") y/o "desesperanzado con respecto al futuro". Se les preguntaban las principales razones de dichos sentimientos y cómo se habían sentido antes de octubre de 2001.

Como puede verse en el cuadro 11, en ambas dimensiones la encuesta indica una incidencia elevada y creciente de desaliento y pesimismo entre los encuestados (en su mayoría jefes de hogar). En el cuadro 12 se presenta mayor evidencia sobre la fuente de este desánimo/pesimismo entre los que informaron tener tales sentimientos. Las "razones económicas" prevalecen sobre las demás razones.

Entre los que informaron sentirse desanimados ahora pero no antes de octubre de 2001, se trató de identificar si este cambio estaba asociado con la situación de empleo del encuestado. Como se muestra en el cuadro 13, no parece ser así. De igual manera, no se encontró ningún patrón asociado con la distribución del ingreso. Estos hallazgos sugieren un impacto generalizado de la crisis económica sobre el estado emocional de la población.

\section{Protestas sociales}

La encuesta confirma un considerable aumento de la participación individual en diferentes formas de protesta social, que pasó del $7.6 \%$ antes de octubre de 2001 al 16.2\%. Es interesante señalar que la participación es mayor a medida que se pasa a los quintiles de ingreso más ricos. Esto concuerda con otro resultado de la encuesta: la forma más popular de protesta fue el cacerolazo (participó el 9.2\%) — una forma de protesta que primó en los grandes centros urbanos durante el primer trimestre de $2002 .{ }^{12}$

La violencia parecería ser factor importante en el período posterior a la crisis. Como se muestra en el

${ }^{12}$ Los grupos de menores ingresos participaron mayormente en piquetes o bloqueo de rutas.

Argentina: Cambios de estado emocional (Porcentajes)

\begin{tabular}{lcccc}
\hline & \multicolumn{2}{c}{ Se siente desanimado } & \multicolumn{2}{c}{ Siente desesperanza sobre el futuro } \\
\cline { 2 - 5 } & Ahora & Antes de octubre de 2001 & Ahora & Antes octubre de 2001 \\
\hline Nunca & 26.8 & 47.8 & 21.1 & 45.5 \\
A veces & 34.2 & 37.3 & 33.2 & 36.2 \\
Frecuentemente & 24.8 & 9.6 & 27.5 & 11.3 \\
Siempre & 13.5 & 4.0 & 16.7 & 4.8 \\
Sin respuesta & 0.6 & 1.3 & 1.5 & 2.2 \\
Total & 100 & 100 & 100 & 100 \\
\hline
\end{tabular}


CUADRO 12

Argentina: Razones del estado emocional (Porcentajes)

\begin{tabular}{lrr}
\hline & $\begin{array}{c}\text { Se siente } \\
\text { desalentado }\end{array}$ & $\begin{array}{c}\text { Siente desesperanza } \\
\text { respecto al futuro }\end{array}$ \\
\hline No encuentra trabajo & 11.3 & 12.7 \\
Dificultades en el trabajo & 1.6 & 1.6 \\
Razones económicas & 53.4 & 54.0 \\
Razones de salud & 4.8 & 3.0 \\
Algo difícil de explicar & 16.1 & 15.4 \\
Otras razones & 12.8 & 13.4 \\
& & 100.0 \\
Total & 100.0 &
\end{tabular}

CUADRO 13

Argentina: Estado de empleo de los que informan desaliento creciente

\begin{tabular}{lr}
\hline Situación actual & $\%$ \\
\hline Empleado & 46.4 \\
Desempleado & 14.5 \\
Jubilado & 15.1 \\
Estudiante & 3.4 \\
Ama de casa & 10.4 \\
Otros inactivos & 10.1 \\
& \\
Total & 100.0 \\
\hline
\end{tabular}

CUADRO 14

Argentina: Participación en protestas sociales

(\% de hogares participantes)

\begin{tabular}{lccccccc}
\hline Quintiles & I & II & III & IV & V & Total & Total antes de octubre 2001 \\
\hline & 11.4 & 12.6 & 17.5 & 17.5 & 22.0 & 16.2 \\
\hline
\end{tabular}

cuadro 15 , el $20 \%$ de los hogares informó haber sido víctima del delito o la violencia en los seis meses previos. Es interesante observar que si bien es cierto que el grupo de mayores ingresos es el que informa la incidencia más alta de delitos o violencia, no existe una correlación simple entre victimización y nivel de ingresos.

Los datos concuerdan con la información recopilada por la Dirección Nacional de Política Criminal para el Gran Buenos Aires, Rosario y Córdoba, que indica que durante el año 2000 el $13 \%$ de los entrevistados había sido víctima de algún tipo de delito (Dirección Nacional de Política Criminal, 2000). La
CUADRO 15

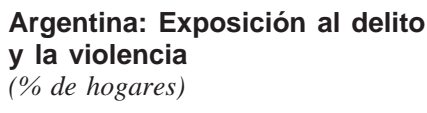

Argentina: Exposición al delito y la violencia

(\% de hogares)

\begin{tabular}{ccccccc}
\hline Quintiles & I & II & III & IV & V & Total \\
\hline & 19.7 & 22.9 & 14.5 & 19.9 & 25.3 & 20.4 \\
\hline
\end{tabular}

información no muestra diferencias significativas entre los diferentes grupos socioeconómicos, pero sí que los grupos de mayores ingresos tienden a informar más la ocurrencia de tales hechos (particularmente debido al robo de automóviles).

\section{III}

\section{Estrategias de supervivencia}

Esta tercera sección del trabajo se concentra en las estrategias adoptadas por los hogares para hacer frente a la crisis. Se comienza con una descripción general de los principales tipos de estrategias que los hogares emplean. Se han agrupado en tres categorías i) estrategias adaptativas de los hogares; ii) estrategias activas de los hogares, y iii) estrategias vinculadas con redes sociales
(Loskin y Yemtsov, 2001). Se sigue con un análisis de los componentes de cada categoría y se identifican los patrones que indican que ciertas estrategias tienden a ser adoptadas por diferentes tipos de hogares (por ejemplo, según niveles de ingresos). Vale aclarar que éste es un primer paso exploratorio de un posterior análisis multivariado sobre las estrategias de supervivencia. 


\section{Una visión general}

Como ya se indicó, en la encuesta se preguntó sobre las estrategias que los hogares usaban en forma más frecuente como reacción ante el empeoramiento de las condiciones económicas experimentado durante los ocho meses previos.

El primer conjunto de estrategias, que se han agrupado bajo el título de estrategias adaptativas de los hogares, incluye las de aquellos que respondieron a la crisis cambiando sus patrones de consumo, incluido el uso de varios tipos de servicios. El segundo conjunto de estrategias, al que se dio el título de estrategias activas de los hogares, involucra un mayor uso de los activos físicos, financieros y humanos de los que el hogar dispone. Entre ellas se cuentan: agregar nuevos trabajadores, trabajar más horas, vender bienes, usar ahorros, pedir prestado, emigrar. El último grupo de estrategias, las vinculadas con redes sociales, incluye aquellas que dependen de la asistencia prestada por amistades, familia, organizaciones no gubernamentales (ONG) o el gobierno.

En el cuadro 16 se resume la información clave referida al uso de estas diferentes estrategias por parte del total de hogares, y específicamente, por el grupo de familias que experimentó una reducción en el ingreso del hogar.
Un primer aspecto que cabe señalar es que casi todos los hogares, sin que importe si han tenido un cambio en su ingreso, informaron haber modificado sus patrones de consumo en respuesta a la crisis económica. Esto se cumple en toda la distribución del ingreso (cuadro 17). Efectivamente, las familias argentinas se han adaptado a la crisis económica cambiando sus patrones de consumo.

Además, con la excepción de las estrategias activas, que tienden a ser usadas de manera más intensa por los hogares que sufrieron la reducción de sus ingresos, no se observa ningún patrón marcado entre los dos grupos cuando se considera el uso de este amplio conjunto de estrategias de supervivencia. Sin embargo, si se concentra la atención en los grupos de ingresos (cuadro 17), al margen de si han experimentado reducción en sus ingresos o no, resulta evidente que el uso de las estrategias vinculadas con las redes sociales presenta una fuerte correlación con los niveles de ingreso.

Como se verá a continuación, más allá de estos patrones generales, pueden observarse diferencias significativas entre los distintos grupos una vez que se desagregan las categorías globales.

\section{Estrategias de adaptación de los hogares}

El cuadro 18 resume el tipo de cambios realizados por los hogares en sus patrones de gasto. En particular,

CUADRO 16

Argentina: Uso de estrategias por hogares

\begin{tabular}{lcc}
\hline & \% de hogares que usa & $\begin{array}{c}\text { De los hogares que informan reducción } \\
\text { de ingreso, } \% \text { que usa }\end{array}$ \\
\hline i) Estrategias adaptativas & 98.2 & 99.1 \\
ii) Estrategias activas & 37.3 & 45.0 \\
iii) Estrategias de redes sociales & 32.7 & 36.4 \\
No usa ninguna de estas estrategias & 1.5 & 0.8 \\
Usa por lo menos una de ellas & 98.5 & 99.2 \\
Usa por lo menos dos de ellas & 54.7 & 61.6 \\
Usa todas ellas & 13.6 & 17.8 \\
\hline
\end{tabular}

CUADRO 17

Argentina: Uso de estrategias por quintiles de ingresos ${ }^{a}$

(\% de hogares de los grupos de ingresos que usan)

\begin{tabular}{|c|c|c|c|c|c|c|c|}
\hline & & I & II & III & IV & $\mathrm{V}$ & Total \\
\hline i) & Estrategias adaptativas & 99.8 & 98.1 & 98.9 & 97.3 & 96.6 & 98.2 \\
\hline ii) & Estrategias activas & 47.0 & 37.7 & 36.1 & 30.5 & 30.7 & 36.4 \\
\hline & Estrategias de redes sociales & 55.5 & 39.1 & 38.4 & 24.2 & 12.0 & 33.8 \\
\hline
\end{tabular}

a Los quintiles de esta sección están construidos con la misma cantidad de hogares en cada quintil, ordenados por ingreso familiar ajustado según tamaño del hogar (usando adultos equivalentes), y excluyendo a los hogares con información de hogares incompleta. 
Argentina: Uso de estrategias adaptativas por quintiles de ingresos

\begin{tabular}{|c|c|c|c|c|c|c|}
\hline Cambios en los patrones de consumo & I & II & III & IV & $\mathrm{V}$ & Total \\
\hline \multicolumn{7}{|l|}{ Alimentos } \\
\hline Reducción de consumo & 90.4 & 83.1 & 73.2 & 69.0 & 59.1 & 74.9 \\
\hline Reemplazo por productos más baratos & 97.6 & 95.4 & 92.5 & 91.5 & 84.8 & 92.3 \\
\hline \multicolumn{7}{|l|}{ Otros bienes } \\
\hline Reducción de consumo & 90.5 & 87.7 & 81.5 & 76.8 & 68.3 & 81.0 \\
\hline Reemplazo por productos más baratos & 89.5 & 89.3 & 80.4 & 80.2 & 76.6 & 83.2 \\
\hline Compra de productos de segunda mano & 51.7 & 40.2 & 34.8 & 33.0 & 24.3 & 36.7 \\
\hline Prescindencia/reducción en el uso de servicio doméstico ${ }^{\mathrm{a}}$ & $\ldots$ & $\ldots$ & 43.0 & 27.8 & 40.5 & 37.9 \\
\hline
\end{tabular}

a Estimado sólo para los hogares que tenían esta opción y sólo cuando se observó un número de casos suficiente.

evidencia la reducción de los niveles de consumo y la sustitución de productos por otros de menor costo. Se distingue entre productos esenciales (alimentos) y no esenciales (otros).

En términos generales, puede verse en el cuadro 18 que todas las familias han reaccionado a la crisis económica a través de una combinación de reducción del consumo y reemplazo por bienes de menor costo, tanto en artículos esenciales como no esenciales. ${ }^{13} \mathrm{No}$ obstante, es posible observar dos patrones. Primero, no debe sorprender que los grupos de mayores ingresos parecerían haber evitado tales cambios en una mayor medida. Esto es particularmente cierto en lo que respecta a los alimentos, donde es notable la diferencia entre quintiles de ingresos. Segundo, la sustitución por productos de menor costo (o productos de segunda mano, cuando es posible) parece ser más generalizada que las reducciones en los niveles de consumo. En otras palabras, la información sugiere que las familias han tratado de proteger el consumo de alimentos tanto como fuera posible, reduciendo el consumo de otros bienes y cambiando por productos de menor costo. Tampoco sorprende observar que los grupos de menores ingresos parecerían haber tenido menor éxito en este sentido.

La encuesta también nos permite observar cambios en el uso de diferentes tipos de servicios y el acceso a ellos (específicamente, educación, salud y servicios públicos).

Una cuestión que merece particular atención -y respecto a la cual se está llevando a cabo un análisis por separado, usando diversas fuentes de información- es si existe evidencia de mayor deserción escolar como resultado de la crisis económica. La encuesta proporciona algunas indicaciones preliminares que acusan una evidencia limitada de dicho efecto. En el cuadro 19 se incluyen tres grupos etarios: 6 a 12 años, 13 a 15 años y 16 a 18 años de edad. En la segunda columna aparecen estimaciones aproximadas de las tasas de cobertura para cada grupo, sin referencia al nivel de escolarización. Con todas sus limitaciones, estos datos confirman las tasas observadas tradicionalmente a través de las encuestas de hogares y no presentan señales de reducción en las tasas de cobertura.

Además, se analizó si existía alguna indicación de deserción escolar durante los primeros meses del año lectivo 2002 entre los niños de los que se informó que no asistían a la escuela. Se les preguntó a los jefes de hogar si alguien del hogar (sin identificar de quién se trataba) ${ }^{14}$ había abandonado o postergado su asistencia escolar desde octubre de 2001. En la tercera columna se informa del porcentaje de niños en el grupo etario correspondiente que no asisten a la escuela y pertenecen a una familia en la que existe por lo menos un miembro que abandonó o postergó el inicio de las clases. Por supuesto, esto puede significar que se sobreestime la presencia de desertores si existe más de un miembro del hogar que desertó o postergó comenzar las clases (incluyendo la educación superior) y, en este sentido, es una estimación de la máxima magnitud potencial del efecto.

\footnotetext{
${ }^{13} \mathrm{El}$ IPC aumentó en $30 \%$ durante los primeros seis meses de 2002. El aumento de precios de los alimentos fue superior (39\%) al promedio. Usando las participaciones de gasto del quintil inferior derivadas de la encuesta de consumo de 1997, estimamos que en este período el costo de vida para los grupos más pobres de la sociedad aumentó un $45 \%$.
}

\footnotetext{
14 De esta manera se evitaba el efecto "vergüenza" que podría haber llevado a una subinformación de los casos de deserción escolar, a cambio de perder información sobre quién era, exactamente, el miembro del hogar involucrado.
} 
Argentina: ¿Las familias han retirado hijos de la escuela?

\begin{tabular}{lccc}
\hline & \multicolumn{2}{c}{ Concurren a la escuela (\% total del grupo) } & \multicolumn{2}{c}{$\begin{array}{c}\text { Estimación de deserción } \\
\text { Trupo etario }\end{array}$} & Área urbana & Área rural & 92.1 \\
\cline { 2 - 4 } 6 a 12 años & 97.0 & 97.3 & 90.9 \\
13 a 15 años & 92.5 & 92.5 & 0.6 \\
16 a 18 años & 80.2 & 81.1 & 60.1 \\
\hline
\end{tabular}

Lo primero que cabe señalar es que, dado el número muy reducido de casos involucrado, las estimaciones no son suficientemente precisas (es decir, tienen un alto coeficiente de variación). Teniendo esto en cuenta, se observa que no existe evidencia de deserción escolar en los grupos de 6 a 12 años o de 13 a 15 años y que hay una presencia reducida (pero no significativa desde el punto de vista estadístico) de desertores escolares en el grupo de 16 a 18 años.

Como prueba de coherencia hemos verificado, para este último grupo, su ubicación dentro de la distribución de ingresos de los hogares. Como lo indica el cuadro 20, los pocos casos de aparentes desertores pertenecen en su mayoría a los deciles inferiores de la distribución, lo que ofrece algún nivel adicional de seguridad con respecto a la evidencia.

La limitada evidencia sobre deserción escolar como resultado de la crisis podría indicar que las familias se esfuerzan por proteger lo que es quizás el tipo de inversión más importante que pueden hacer en sus hijos bajo difíciles circunstancias (De Ferranti, Perry y otros, 2000). No obstante, como sugiere la evidencia proveniente de otras crisis, los efectos negativos pueden no sentirse en forma inmediata sino varios meses después del comienzo de la crisis (por ejemplo, podrían hacerse sentir en los niveles de matriculación escolar en 2003). Además, los datos de la encuesta no permiten identificar el número de niños que no asisten a clase de manera regular, que quizás en muchos casos sea un tipo de ajuste más prevaleciente.

En el cuadro 21 se proporciona alguna evidencia adicional sobre si las familias hicieron ajustes que afectaron la educación de sus hijos. La proporción de familias que cambió de escuela (a escuelas privadas menos costosas o de la escuela privada a la escuela pública) es muy reducida, lo que sugiere una resistencia a realizar dichos cambios, inclusive en el contexto de un marcado deterioro económico.

Por otro lado, una proporción muy grande de los hogares (particularmente en los grupos de ingresos más
CUADRO 20

Argentina: Deserciones escolares (Porcentajes)

\begin{tabular}{lc}
\hline Quintiles & Deserciones en el grupo de $16-18$ años \\
\hline I & 53.9 \\
II & 23.3 \\
III & 6.3 \\
IV & 16.6 \\
V & 0 \\
\hline
\end{tabular}

CUADRO 21

\section{Argentina: Ajustes en los gastos de educación \\ (\% de hogares)}

Reemplazaron escuela privada por escuela pública

Cambiaron a escuelas privadas menos costosas

bajos) ${ }^{15}$ informó haber reducido la compra de materiales escolares - con efectos aún no determinados sobre la calidad de la escolarización. Las dificultades experimentadas por varias provincias para mantener los niveles de gasto sugieren que la presencia de restricciones por el lado de la oferta puede ser más seria que por el de la demanda, por lo menos en esta etapa temprana del proceso.

En el caso de los servicios de salud, existe evidencia de impactos negativos severos desde comienzos del año 2002, tanto en cuanto a dificultades dentro del sistema de obras sociales como en el sistema de salud pública. La información recogida a través de la encuesta confirma las tendencias identificadas antes.

Aproximadamente el 12\% de los individuos experimentó algún cambio en su cobertura de salud (cuadro 22). Más del $60 \%$ perdió totalmente su cobertura — con

${ }^{15}$ Casi el $90 \%$ de los hogares en el quintil de ingresos inferiores informó sobre reducciones en la compra de materiales escolares, mientras que sólo $43 \%$ de los del quintil más alto informó lo mismo. 
CUADRO 22

Argentina: Cambios en la cobertura médica (\% de los individuos que experimentaron un cambio de cobertura)

\begin{tabular}{lcccccc}
\hline Quintiles & I & II & III & IV & V & Total \\
\hline $\begin{array}{l}\text { Pérdida de toda forma } \\
\text { de cobertura }\end{array}$ & 76.0 & 61.1 & 78.6 & 52.6 & 33.6 & 61.4 \\
Cambio de cobertura & 24.0 & 38.9 & 21.4 & 47.4 & 66.4 & 38.7 \\
\hline
\end{tabular}

una concentración mayoritaria en los grupos de menores ingresos. El $40 \%$ de aquellos que experimentaron un cambio en su cobertura de salud pasó a una cobertura menor. Casi la mitad de aquellos que antes tenían una cobertura doble (obra social y prepaga) ahora solamente mantienen su cobertura primaria (obra social). También se observa cambio de una cobertura privada completa a una cobertura para emergencias únicamente.

La pérdida de cobertura de salud está conduciendo a que sea creciente el número de personas que dependen del uso de las instalaciones de salud públicas. De los hogares que informaron haber reemplazado la cobertura privada por los servicios públicos como reacción a la crisis económica, el $60 \%$ ha experimentado alguna forma de pérdida o limitación de la cobertura ofrecida por sus prestadores. En términos más específicos, el $16 \%$ perdió totalmente su cobertura, $5 \%$ tiene deudas pendientes que no les permite usar su cobertura, $13 \%$ no puede costear los copagos, $8 \%$ experimentó una reducción de los servicios ofrecidos por los aseguradores, y el $13 \%$ son jubilados que tienen dificultades con los servicios ofrecidos por el Instituto Nacional de Servicios Sociales para Jubilados y Pensionados (PAMI). ${ }^{16}$

En términos generales, la encuesta determinó que las familias han reducido de una u otra manera los servicios de salud como consecuencia de la crisis económica. Vale la pena mencionar algunos resultados. Casi el $23 \%$ de los hogares informó que por lo menos un miembro había experimentado la falta de acceso a servicios de salud. Tres cuartos de ellos dieron como razón la "falta de dinero" para pagar los medicamentos $(44 \%)$, los costos de transporte $(25 \%)$ y las cuotas (5\%). Más específicamente, el $37 \%$ de los hogares con niños menores de doce años informaron haber reducido la frecuencia con la que llevan a sus hijos a los controles médicos. Casi el $45 \%$ de estos hogares pertenecen al quintil más bajo de distribución de los in-

\footnotetext{
16 PAMI es el seguro nacional de salud para esos grupos.
}

CUADRO 23

Argentina: Cambios en el uso de servicios de transporte

\begin{tabular}{|c|c|c|c|c|c|c|}
\hline & I & II & III & IV & V & Total \\
\hline $\begin{array}{l}\text { Auto/taxis por } \\
\text { transporte público } \\
\text { Auto/taxi/transporte } \\
\text { público por }\end{array}$ & 57.4 & 56.7 & 47.8 & 45.5 & 2.4 & 46 \\
\hline bicicleta/caminata & 73.9 & 62.5 & 61.3 & 49.0 & 33.5 & 55.4 \\
\hline
\end{tabular}

gresos. Pero este efecto también se sintió en el segundo $(29 \%)$ y tercer (18\%) deciles.

Reducir el uso de servicios de utilidad pública aparece como otra de las estrategias de los hogares. En forma típica, esto significa ya sea demorar o dejar de pagar el servicio (con el riesgo de desconexión) o directamente pedir su interrupción. Entre el 30\% y el $50 \%$ de los hogares informa de demoras en los pagos. El porcentaje de hogares que informó haber sido desconectado de diferentes servicios (electricidad, gas, agua, teléfono, cable, Internet) va del $2 \%$ (en el caso del suministro de agua) al 14\% para la televisión por cable. En la mayor parte de los casos, la principal razón fue la falta de pago.

De igual manera, los hogares han comenzado a utilizar más los medios de transporte de menor costo, como muestra el cuadro 23. Más de la mitad de los hogares que antes usaban sólo transporte público informan ahora haber aumentado el uso de la bicicleta o la caminata. Se observa un cambio similar desde los automóviles y taxis al transporte público.

Estas cifras son consistentes con las que surgen de los datos oficiales sobre el uso de los servicios públicos ${ }^{17}$ que indican, para los primeros cinco meses de 2002, una reducción de $22 \%$ en el número de pasajeros en los ferrocarriles urbanos, $9 \%$ en subterráneos y $14 \%$ en los servicios de ómnibus metropolitanos (Estadísticas de Servicios Públicos, 2002).

\section{Estrategias activas de los hogares}

El cuadro 24 resume algunas de las medidas activas adoptadas por los hogares en respuesta a la crisis económica. Sólo en el 13\% de los hogares se pudo observar el ingreso de un nuevo miembro a la fuerza de trabajo.

\footnotetext{
${ }^{17}$ Estadísticas de Servicios Públicos (2002). Estas estadísticas son recopiladas en forma mensual por el INDEC y constituyen la base para su Indicador Sintético de Servicios Públicos (ISSP).
} 
Estrategias en el mercado laboral

Suman nuevos trabajadores al mercado laboral

13.4

14.8

Trabajan más horas

Tiempo

Aumentan la producción casera

Prescinden/reducen servicio doméstico ${ }^{\mathrm{a}}$

Migración

59.9

35.3

4.1

a El porcentaje aquí se refiere a hogares a los que la categoría es aplicable.

CUADRO 25

Argentina: Estrategias activas de los hogares por nivel de ingresos

\begin{tabular}{|c|c|c|c|c|c|c|}
\hline Quintiles & I & II & III & IV & $\mathrm{V}$ & Total \\
\hline \multicolumn{7}{|l|}{ Estrategias en el mercado laboral } \\
\hline Suman nuevos trabajadores al mercado laboral & 28.0 & 16.8 & 12.2 & 6.2 & 1.4 & 12.9 \\
\hline Trabajan más horas & 11.4 & 15.6 & 16.3 & 11.5 & 13.4 & 13.7 \\
\hline \multicolumn{7}{|l|}{ Tiempo } \\
\hline Aumentan la producción casera & 74.4 & 73.0 & 62.6 & 52.5 & 43.2 & 61.1 \\
\hline Prescinden/reducen servicio doméstico ${ }^{\mathrm{a}}$ & & & 43.0 & 27.8 & 40.5 & 37.9 \\
\hline Migración & 4.3 & 2.3 & 6.0 & 5.1 & 4.6 & 4.5 \\
\hline
\end{tabular}

a El porcentaje aquí se refiere a hogares a los que la categoría es aplicable.

Esto es particularmente importante en el grupo de ingresos más bajos (cuadro 25). Típicamente se trata de un trabajador secundario: en 50\% de los casos es el hijo o hija y en el $25 \%$ de los casos es el cónyuge de la persona informada como jefe/jefa del hogar. Sin embargo, la eficacia de esta estrategia parece ser algo limitada. Poco más del $13 \%$ de estos nuevos ingresantes al mercado laboral logra encontrar trabajo. Los que tienen éxito tienden a no ser los más pobres entre los ingresantes.

Trabajar más horas aparece como otra estrategia adoptada por todos los grupos de ingresos. No obstante, los que parecen tener éxito en este esfuerzo por aumentar el número de horas que trabajan parecen ser en su mayoría los trabajadores asalariados con trabajos permanentes, particularmente en el comercio.

En paralelo con estos cambios en las estrategias de empleo, es posible observar una tendencia a reemplazar el ocio por el trabajo en el hogar como manera de hacer frente a la crisis. Vale la pena mencionar dos ejemplos. Primero, alrededor del $60 \%$ de los hogares informó haber aumentado el tiempo que dedica a la preparación de "bienes producidos en el hogar" (por ejemplo, comidas) en reemplazo de productos comprados. Como se muestra en el cuadro 25, esta estrategia es ampliamente usada por los grupos de ingresos más bajos. En segundo lugar, entre los hogares que emplean servicio doméstico hay una notable reducción de su uso.

La encuesta también indagaba sobre la migración de algún miembro del hogar desde octubre de 2001 o si alguien en el hogar estaba considerando esta opción. En promedio, algo más del $4 \%$ de los hogares informaron tener por lo menos un miembro que había emigrado, un porcentaje más o menos uniforme entre los diferentes grupos de ingresos. ${ }^{18}$ Las principales razones de la migración incluyen "falta de trabajo" (58\%) y búsqueda de una mejor calidad de vida (15\%). Por otro lado, más del $20 \%$ de los hogares informaron que por lo menos un miembro de la familia estaba considerando emigrar. La principal opción que era considerada (80\% de los hogares) era la migración a otro país

\footnotetext{
${ }^{18}$ Se excluyen los que emigraron como consecuencia de matrimonio, enfermedad u otras razones familiares.
} 


\begin{tabular}{|c|c|c|c|c|}
\hline & \multicolumn{2}{|c|}{$\%$ de hogares que usa } & \multicolumn{2}{|c|}{$\begin{array}{c}\text { De los hogares que informaron } \\
\text { una reducción de ingresos, } \\
\% \text { que usa }\end{array}$} \\
\hline & Ahora & Antes de oct. 2001 & Ahora & Antes de oct. 2001 \\
\hline Venta de activos & 3.7 & 1.1 & 5.5 & 1.0 \\
\hline Uso de ahorros & 5.1 & 3.0 & 7.1 & 4.9 \\
\hline Préstamos bancarios & 1.7 & 2.4 & 1.3 & 3.1 \\
\hline Compra con pago diferido & 7.3 & 5.1 & 9.7 & 6.6 \\
\hline
\end{tabular}

CUADRO 27

\section{Argentina: Uso de estrategias financieras por nivel de ingresos}

\begin{tabular}{lcccccc}
\hline Quintiles & I & II & III & IV & V & Total \\
\hline $\begin{array}{l}\text { Venta de activos } \\
\text { Uso de ahorros }\end{array}$ & 5.9 & 3.7 & 3.3 & 2.7 & 1.1 & 3.3 \\
$\begin{array}{l}\text { Préstamos bancarios } \\
\begin{array}{l}\text { Compra con pago } \\
\text { diferido }\end{array}\end{array}$ & 0.9 & 3.6 & 1.8 & 0.6 & 2.0 & 1.8 \\
& 14.6 & 13.1 & 9.5 & 2.3 & 0.7 & 8.0 \\
\hline
\end{tabular}

- particularmente entre los grupos de mayores ingresos de la Ciudad de Buenos Aires.

Desde el comienzo de la crisis, una mayor proporción de los hogares está usando sus ahorros, vendiendo activos o tomando prestado como manera de sostener el consumo (cuadro 26).

Quizás el hecho más interesante que se observa sea el grado en que los hogares de menores ingresos dependen como estrategia de supervivencia del uso de crédito informal otorgado por los comercios del barrio (la compra al fiado) que les permite diferir el pago (cuadro 27).

\section{Estrategias vinculadas con redes sociales}

En el cuadro 28 se resumen algunos de los principales indicadores sobre el uso de redes sociales por parte de los hogares. En el cuadro 29 puede verse información adicional desagregada por grupo de ingresos. Globalmente, se observa que más de un tercio de los hogares informa beneficiarse, por lo menos en un aspecto, del acceso a una red de contención social. El uso de estas estrategias es algo mayor en los hogares que experimentaron caídas nominales negativas en sus ingresos (cuadro 28) y significativamente mayor en los hogares del extremo inferior de la distribución de ingresos (cuadro 29).

La encuesta brinda alguna evidencia de que las redes sociales se utilizan de modo diferente a lo largo de la distribución del ingreso. Efectivamente, si se compara la distribución de los hogares que informan haber recibido o dado asistencia a personas que no habitan en su propio hogar, puede observarse que los hogares del quintil inferior tienden a ser receptores netos mientras que los del quintil superior tienden a ser dadores netos.

A través de preguntas retrospectivas, se pudo identificar cambios en el acceso a redes sociales o su uso desde octubre de 2001. Si bien en la encuesta se registra un aumento de diferentes formas de uso de las redes sociales, los incrementos mayores se dan en el uso del trueque y la participación en actividades comunitarias.

La intensidad del uso del trueque en los grupos de bajos ingresos se hace patente en el hecho de que el ingreso per cápita de los hogares que lo utilizan es menor que el del total de la población (143 pesos contra 214 , y 183 pesos contra 264, con y sin ajuste por adulto equivalente, respectivamente). Los hogares que recurren al trueque tienden a tener más miembros desempleados (43.3\% contra $22.4 \%$ ) y más trabajadores temporarios ( $42 \%$ contra $23 \%$ ), lo que es prueba adicional de su vulnerabilidad.

Un patrón similar se observa en los hogares que informaron recibir algún tipo de asistencia oficial. Sus ingresos per cápita están por debajo del promedio (122.7 pesos y 97.62 pesos con y sin ajuste por adulto equivalente), tienen una mayor incidencia de desempleo (65\% de estos hogares tiene un miembro desocupado) y, en el caso de los miembros que están ocupados, tienden a tener más trabajos temporarios (80\%). 
Asistencia de otras personas que no viven en la casa

16.3

10.7

6.9

20.9

11.1

37.0

14.6
17.0

13.7

7.5

26.4

15.2

42.5

19.9

a Un mayor porcentaje de hogares (22\%) informó que brindaba asistencia a personas que no habitaban en el mismo hogar

b Las actividades comunitarias incluyen las siguientes categorías: comedores escolares, comedores comunitarios, compras comunitarias de mercadería, talleres comunitarios, recaudación de fondos, guarderías comunitarias, entre otras.

Argentina: Uso de estrategias vinculadas con redes sociales, por nivel de ingresos

\begin{tabular}{|c|c|c|c|c|c|c|}
\hline & I & II & III & IV & V & Total \\
\hline \multicolumn{7}{|l|}{ Asistencia de otras personas } \\
\hline $\begin{array}{l}\text { que no viven en la casa } \\
\text { Asistencia a otras personas }\end{array}$ & 26.4 & 15.5 & 20.9 & 15.8 & 7.3 & 17.1 \\
\hline que no viven en la casa & 10.0 & 19.7 & 17.1 & 21.2 & 37.9 & 21.2 \\
\hline Préstamos de amigos y familia & 21.2 & 15.7 & 10.6 & 5.8 & 3.0 & 11.3 \\
\hline Planes sociales & 18.5 & 6.1 & 7.5 & 1.3 & 0.1 & 6.7 \\
\hline Participación en actividades comunitarias & 29.4 & 23.6 & 22.8 & 15.7 & 15.7 & 21.4 \\
\hline Trueque & 20.2 & 15.4 & 11.7 & 5.5 & 3.9 & 11.3 \\
\hline
\end{tabular}

\section{IV}

\section{A modo de conclusión}

El trabajo presenta varias evidencias de la magnitud de los costos sociales de la crisis económica argentina y sus efectos adversos sobre el bienestar a lo largo de todos los deciles de la distribución del ingreso. Los datos a nivel de hogar confirman la imagen negativa obtenida a partir del análisis de los indicadores macroeconómicos disponibles desde los comienzos del año.
Los hogares están usando variadas estrategias para responder a una intensa crisis. Los limitados datos sobre consumo recopilados no permiten determinar en qué medida las reducciones en los niveles de ingreso están produciendo formas de privación extremas (es decir, en qué medida las estrategias de supervivencia permiten a las familias evitar las restricciones 
importantes). No obstante, la magnitud de las perturbaciones y las limitaciones en la eficacia de los diferentes mecanismos de supervivencia que se han identificado hacen pensar que los efectos sobre el bienestar son efectivamente muy serios. La evidencia sugiere efectos más marcados en el uso de los servicios de salud que en educación, conclusión que se está analizando en más detalle utilizando fuentes de información adicionales.

Una rápida comparación con lo sucedido en otros países que han experimentado perturbaciones económicas de una magnitud parecida muestra algunas similitudes muy interesantes, así como algunas diferencias específicas. El análisis realizado por Fallon y Lucas (2002) de los efectos sobre los mercados laborales ${ }^{19}$ señala un patrón de crecientes índices de desempleo abierto e importantes cambios en la composición sectorial del empleo (marcadas reducciones en la construcción y la manufactura, en esta última a medida que el sector empresarial se ve afectado por los mayores costos de los materiales importados, las dificultades para la obtención de crédito y la creciente carga de la deuda en moneda extranjera) similar al observado en el caso de la Argentina posterior a la convertibilidad.

La evidencia sobre el impacto distributivo de las crisis muestra algunas diferencias entre los distintos países. Thomas, Frankenberg y otros (1999) estimaron que en el caso de Indonesia se produjo una declinación en los gastos per cápita en los quintiles superior e inferior de la distribución que fue mayor en el caso de los hogares ubicados en un punto medio de la distribución. En Tailandia, en cambio, se comprueba una débil redistribución de ingresos desde las clases de ingresos medios hacia las de ingresos altos. Los datos analizados en el presente trabajo sugieren que las reducciones en el ingreso familiar en Argentina han afectado más a la clase media.

En lo que hace a las estrategias de supervivencia, al igual que en el caso de Argentina, Frankenberg, Thomas y otros (1999) observan que la asistencia informal de amigos y miembros de la familia fue particularmente importante en Indonesia durante la crisis, pues alrededor de un cuarto de los hogares recibió dicha asistencia. Su valor medio fue considerablemente mayor que el de la asistencia de los servicios formales. Sudarno, Wetterberg y Pritchett (1999) también determinaron en el caso de Indonesia diferencias en las estrategias de supervivencia en función de la distribución del ingreso. Por ejemplo, las familias de clase media respondieron trabajando más horas, reduciendo el consumo, utilizando sus ahorros y vendiendo activos, mientras que los hogares de menores ingresos recurrieron a medidas más drásticas, tales como retirar a sus hijos de la escuela.

Con respecto a este último punto, la evidencia es mixta. Por ejemplo, en México durante la crisis de 1982 las tasas de deserción de la escuela secundaria aumentaron levemente mientras que se reducían los índices de deserción en la escuela primaria. Ambos cambios formaron parte de tendencias de largo plazo para toda la década de 1980 (Lustig, 1998). De igual manera, Adam y Chamberlin (1999) no encontraron evidencia de reducciones significativas de la participación escolar en Tailandia, aunque también reconocieron las dificultades de medición y respuestas demoradas que hemos mencionado. La evidencia sobre Tailandia, no obstante, mostró una brecha entre los índices de deserción escolar de los hogares pobres y no pobres, tal como se pudo determinar (aunque con poca precisión) en el caso de Argentina.

Finalmente, si bien la crisis actual es de magnitud mucho mayor que las experimentadas en el decenio de 1990, las características generales de las estrategias de supervivencia que se observan son muy similares a las halladas por estudios previos de Argentina (Banco Mundial, 2001). Cabe mencionar, en particular, la dependencia de las redes sociales y los cambios en los patrones de consumo.

Los siguientes pasos para continuar este análisis incluyen estudios más detallados del impacto de la crisis sobre los servicios de salud y educación, un análisis más sistemático del uso de diferentes estrategias de supervivencia por tipo de hogar (usando métodos de variables múltiples), una consideración más específica del sector rural —empleando la singular cobertura de esta encuesta - y una atención particular al papel de la migración como estrategia de supervivencia.
${ }^{19}$ Los siguientes son los países analizados y los años de sus crisis,
en orden cronológico invertido: Indonesia (1998), la República de
Corea (1998), Malasia (1998), Tailandia (1997), Argentina (1995), México (1995) y Turquía (1994). 
Bibliografía

Adam, Sarah y Christopher Chamberlin (1999): Thailand Social Monitor. Coping with the Crisis in Education and Health, World Bank Report, No 24467 TH, Washington, D.C.

Banco Mundial (2001): Household Risk, Self-Insurance and Coping Strategies in Urban Argentina, Report, No 22426-AR, Washington, D.C.

(2000): Poor People in a Rich Country: A Poverty Report for Argentina, Report, $\mathrm{N}^{\circ}$ 19992-AR, Washington, D.C.

De Ferranti, David; Guillermo E. Perry, y otros (2000): Securing our Future in a Global Economy, Washington, D.C., Banco Mundial, junio.

Dirección Nacional de Política Criminal (2000): Encuestas de Victimización en Buenos Aries, Rosario, Córdoba y Gran Buenos Aires.

Heckman, James (1979): Sample selection as a specification bias, Econometrica, vol. 47, $\mathrm{N}^{\circ} 1$, enero.

Estadísticas de Servicios Públicos (2002): Información de Prensa, Buenos Aires, Instituto Nacional de Estadística y Censos (INDEC)/Ministerio de Economía.

Fallon, Peter R. y Robert E.B. Lucas (2002): The impact of financial crises on labor markets, household incomes and poverty: a review of evidence, The World Bank Research Observer, vol. $17, \mathrm{~N}^{\circ} 1$, Washington, D.C.
Frankenberg, Elizabeth; Duncan Thomas, y Kathleen Beegle (1999): The Real Costs of Indonesia's Crisis: Preliminary Findings from the Indonesia Family Life Surveys, Labor and Population Program Working Paper Series, No 99-04, Santa Monica California, Rand Corp., marzo.

Loskin, M. y R. Yemtsov (2001): Household Strategies for Coping with Poverty and Social Exclusion in Post-crisis Russia, Policy Research Working Paper, Washington, D.C., Banco Mundial.

Lustig, Nora (2000): Crisis and the poor: socially responsible macroeconomics, Journal of the Latin American and Caribbean Economic Association, vol. 1, No 1, The Brookings Institution Press.

(1998): Mexico: The Remaking of an Economy, Washington, D.C., The Brookings Institution Press.

Sudarno, Sumarto; Anna Wetterberg, y Lant Pritchett (1999): The social impact of the crisis in Indonesia: results from a nationwide Kecamatan survey, East Asia Environment and Social Development Unit, $\mathrm{N}^{\mathrm{o}} 21249$.

Thomas, Duncan; Elizabeth Frankenberg, y otros (1999): Household Budgets, Household Composition and the Crisis in Indonesia: Evidence from Longitudinal Household Survey Data, documento presentado en la Reunión de la Asociación de Población de América (Nueva York). 
